Notfall Rettungsmed 2016 · 19:3

DOI 10.1007/s10049-015-0126-5

Online publiziert: 29. Januar 2016

(c) Springer-Verlag Berlin Heidelberg 2016

CrossMark

\author{
R. Somasundaram ${ }^{1} \cdot$ C. Wrede ${ }^{2}$ \\ ${ }^{1}$ Interdisziplinäre Rettungsstelle und Aufnahmestation, Campus Benjamin \\ Franklin, Charité - Universitätsmedizin Berlin, Berlin, Deutschland \\ ${ }^{2}$ Interdisziplinäres Notfallzentrum, HELIOS Klinikum Berlin-Buch, Berlin, Deutschland
}

\title{
Notfallversorgung von Patienten mit alkoholbedingten Erkrankungen
}

Liebe Leserinnen und Leser,

akute alkoholbedingte Erkrankungen sind im Rettungsdienst und in den Notaufnahmen sehr häufig, die rechtlichen und medizinischen Aspekte der Behandlung werfen dennoch häufig Fragen auf.

\section{》) Alkoholisierte Patienten im Rettungsdienst sind oft schwer einschätzbar}

Alkoholisierte Patienten im Rettungsdienst sind oft nur schwer einschätzbar, da die Menge und der Zeitpunkt der Einnahme meist unbekannt ist, die Anamnese und auch die körperliche Untersuchung Mischintoxikationen ergeben sowie Begleitverletzungen vorliegen können. Gleichzeitig sind die Patienten häufig agitiert.

Im ersten Beitrag geht Sarangi auf die rechtlichen Besonderheiten in der präklinischen Versorgung alkoholisierter Patienten ein, und thematisiert die Einhaltung der medizinischen Standards, die bei alkoholisierten Patienten gleichermaßen wie bei jedem anderen Patienten Anwendung finden müssen, obwohl Anamnese und Befunderhebung oft erschwert sind. Vor diesem Hintergrund weist er besonders auf die Notwendigkeit einer guten Dokumentation hin. Weitere häufig auftretende Herausforderungen sind die Beurteilung der Einsichtsfähigkeit der Patienten und der Umgang mit aggressiven Patienten, die in diesem Artikel aus rechtlicher Sicht beleuchtet werden. Die spezielle recht- liche Situation bei der Anordnung von Blutalkoholentnahmen wird von Jäkel in einem weiteren Beitrag dargestellt. Die Epidemiologie und Pathophysiologie der akuten Alkoholintoxikation sowie die medizinischen Therapiestandards in Notaufnahmen werden von Hans et al. aufgezeigt. In diesem Artikel sowie schwerpunktmäßig in dem Beitrag von Haas wird die Erkennung des Alkoholentzugsdelirs mit den wesentlichen Differenzialdiagnosen dargestellt und die oft schwierige medikamentöse Therapie diskutiert. Eine akute Alkoholintoxikation kann mit Komplikationen wie beispielsweise einer alkoholischen Ketoazidose oder Begleitverletzungen einhergehen, und chronischer Alkoholmissbrauch ist mit Alkoholfolgeerkrankungen und psychiatrischen Erkrankungen assoziiert, auf die von Schilling et al. im letzten Beitrag dieses Leitthemenhefts eingegangen wird. Wir hoffen, dass wir Ihnen mit diesem Heft neue Aspekte des häufigen und relevanten Themas der alkoholbedingten Erkrankungen nahebringen können.

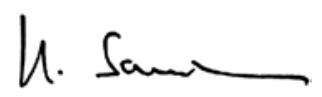

R. Somasundaram

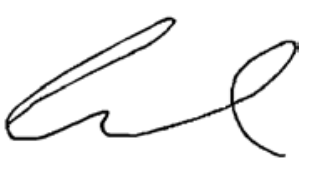

C. Wrede

\section{Korrespondenzadresse}

Prof. Dr. med. C. Wrede

Interdisziplinäres Notfallzentrum, HELIOS Klinikum Berlin-Buch

Schwanebecker Chaussee 50, 13125 Berlin

christian.wrede@helios-kliniken.de

\section{Einhaltung ethischer Richtlinien}

Interessenkonflikt. R. Somasundaram und C. Wrede geben an, dass kein Interessenkonflikt besteht.

Dieser Beitrag beinhaltet keine Studien an Menschen oder Tieren. 